\title{
Data, Models and Computer Vision: Three Hands-on Projects
}

\author{
Valerio Palma
}

Abstract

Advances in information and communication technologies unveil unprecedented opportunities in the documentation and analysis of architectural and urban assets. Nevertheless, the (over-)abundance of data does not always seem sufficient for a thorough understanding. This contribution summarizes three connected research experiences that produced apps and prototypes exploiting geospatial databases, Al for image recognition, and AR based on model tracking, applied to built heritage contexts. The three cases contribute to a reflection on the interpretation of the form of the built environment as a means to produce shared knowledge and operational outcomes.

Keywords

augmented reality, artificial intelligence, digital archives, information modeling.

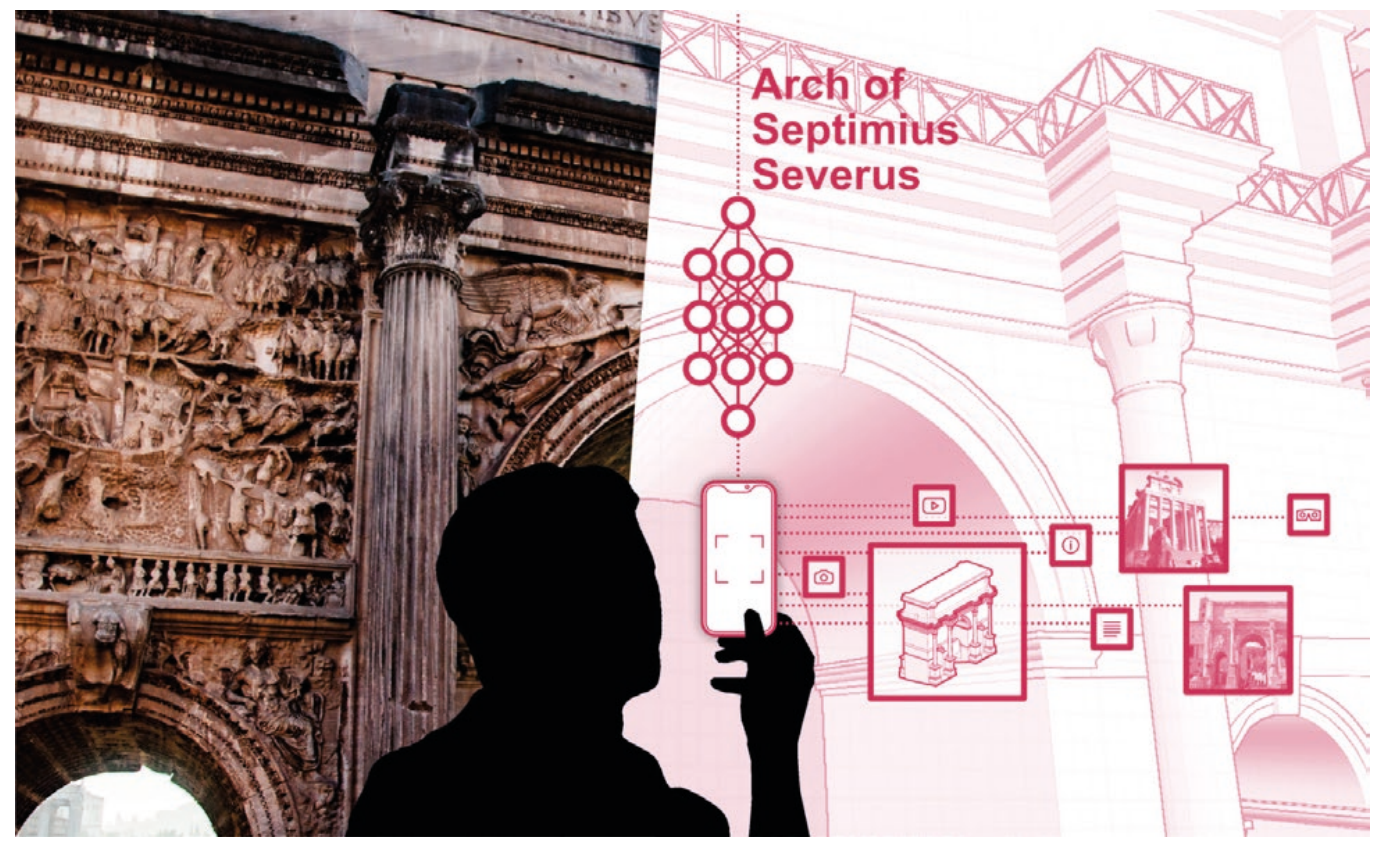




\section{Introduction}

Much of the narrative about technological advancement associates the collection of extensive and precise data with the quality of our understanding of architectural and urban assets. Augmented reality (AR), artificial intelligence (AI), digital survey, and digital modeling nourish this need for information. On the one hand, these rapidly evolving fields allow for unprecedented and fascinating overlaps and synergies. On the other hand, curbing the hype that surrounds them is often needed. New technologies require attention to their fallibility, to the clarity of the purpose for which we use them, and to the generated models, which mediate our understanding of the documented objects. For example, the problems related to the classification of physical assets affect many tools - and in particular to those of Al, capable of manipulating abstract concepts. As data increase, who chooses the used categories, and how can these be adapted to each application that involves the tools?

This contribution collects some 'fieldwork', that is, experiences in prototyping with technologies to which, increasingly, we entrust the interpretation, classification, automatic replication, and visualization of the built space. The projects described constitute the stages of a branched but coherent research path started at the ICEA department of the Università di Padova and carried on at the Politecnico di Torino, through the activities of the FULL laboratory and with the collaboration between the author, Roberta Spallone and Marco Vitali at the Department of Architecture and Design.

The first project concerns the development of a web platform, based on a geospatial database, and designed to guarantee access and interconnection to the multimedia results of architectural and heritage research. The project fostered a reflection on how even the vast digital information topology can benefit from the physical set of sources and their spatial characters. The second project is part of a study on the vaulted atriums of baroque Turin, which produced a catalog of over 70 sites and analyzed the geometric components of the vaults through digital models. The analysis and representation work was integrated with an AR application to visualize the geometric models superimposed against the images of the real vaults. Besides, we designed the system interaction with the web database built during the first cited project. The third project develops a mobile application that allows access to data on monuments by framing a target object with the camera and exploiting machine learning technologies for image recognition. This conjunction between $\mathrm{Al}$ and the built space aims at making cultural heritage more accessible thanks to low-cost, non-perishable, and easy-to-use infrastructures.

The research path is an opportunity for reflecting on the processes that, from techniques for the recognition of spatial features - including AI and AR -, produce a schematic understanding and operational uses of the built form.

\section{First Project: a Flexible Digital Archive}

The first project, entitled Tu-Cult, lasted one year, was carried out at the Università di Padova and was directed by Luigi Stendardo and Andrea Giordano [I]. The project produced a digital infrastructure on which subsequent experiences have relied, and involved several academic and industrial partners and eight researchers in different architecture-related fields including information modeling, geomatics, history, representation. The project featured the study of two churches in Padova, a small sample made significant by the differences between the two cases and conceived as an incremental contribution to a work on other monuments already started by part of the team [Cecchini et al. 20I9].

The team aimed to enhance cultural tourism by means of information and communication technology (ICT). This objective was pursued through three different kinds of outputs: (I) 'data', including documents, raw data, and all the other principal information sources; (2) 'models', that is, 3D models, information modeling, and other data processing products; (3) 'visualization', that is, tools and applications to deliver data and models to the final user through visual interfaces. Therefore, a significant part of the project dealt with the quantity and heterogeneity of the possible interpretations that characterize built heritage - which the 
different outputs and professionals involved were able to grasp - and specifically addressed the role of representation as a means for sharing knowledge [Giordano 20 I7].

The team produced historical and documentary research, detail models and reconstructions of historical phases, BIM models and digital survey models, visualizations in VR and AR. Intending to manage and connect these products, we set up the [cult] platform (fig. I, top left) [2]. It is based on a geospatial database and a set of web services, and makes the project information accessible and connected, even for external applications. The system does not underlie a complex hierarchy - it collects the entries into three simple categories: texts, images, and models - but is based on ease of use and flexibility that have been verified during subsequent projects. The platform was built only with free and open-source software: the web interface was programmed with the Django framework, while the archive is a PostgreSQL database with the PostGIS spatial extension. Metadata are organized based on the Dublin Core standard for resource description [3]. Other technical aspects of the project are described in previous publications [Cecchini et al. 2019].

\section{Second Project: Augmented Reality and the Built Environment}

The second project was started at the Department of Architecture and Design of the Politecnico di Torino. The research explores AR applications to the architectural environment and the possibility, already introduced in the context of cultural heritage, of making digital archives accessible from physical objects. The research team - composed of Roberta Spallone, Marco Vitali and the author - had previously employed AR to connect physical archives and databases, within a project on the Theatrum Sabaudiae, catalog of the Savoy family residences at the end of the eighteenth century [Palma et al. 2018]. The result was an application anchoring small-scale 3D models to images. This experience evolved into a project to share the results of a study on over 70 Turin baroque atria of the seventeenth and eighteenth century, previously conducted by Spallone and Vitali [20 I 7] [4]. Advances in AR allowed a shift in our experiments towards the architectural scale - even if, in early 2019 (the start of the project), commercial AR technologies mainly supported small-scale applications [5].

We developed an app to view models of the geometric interpretation of the atrium vaults (fig. I, bottom).

The tests were conducted on a sample of four atria. The objects tracked for AR activation, were three-dimensional objects, of limited extension with respect to the entire environment, such as the base of columns, or capitals. The targets were first registered as sparse point clouds and finally recorded in the app to enable runtime recognition. Since AR tools use many sensors (such as inertial platforms) to track the changing position of the mobile device, the small-sized targets allow visualizing the entire superimposed vault models with fair accuracy - even when the target is no longer framed. Finally, we verified the compatibility of the documentation on the atria with the [cult] platform, intending to use it as the database to be navigated from the AR view. In this case, we used the Xcode programming interface, a commercial software allowing free development. Other aspects of the project have been explored in previous publications [Palma, Spallone, Vitali 2019].

This is still an ongoing project since the digital archive based on the atria has yet to be produced. Furthermore, in the current state of technological advances, new solutions should be tested to use AR in the built space.

\section{Third Project: Deep Learning for the Recognition of Architecture}

The third project was conducted at the FULL Interdepartmental Center of the Politecnico di Torino [6]. The project aimed to optimize the resources invested in managing $\mathrm{BH}$ and the related information, using ICTs. Many solutions already in use at tourist sites (for example audio guides, or self-guided tours with QR codes) still require expensive and perishable infrastructures and ad hoc projects. We have therefore created a solution for connecting the physical environment to the virtual space of information, documents, and digital models, exploiting the capabilities of Al, the developments of which are starting to show the application 

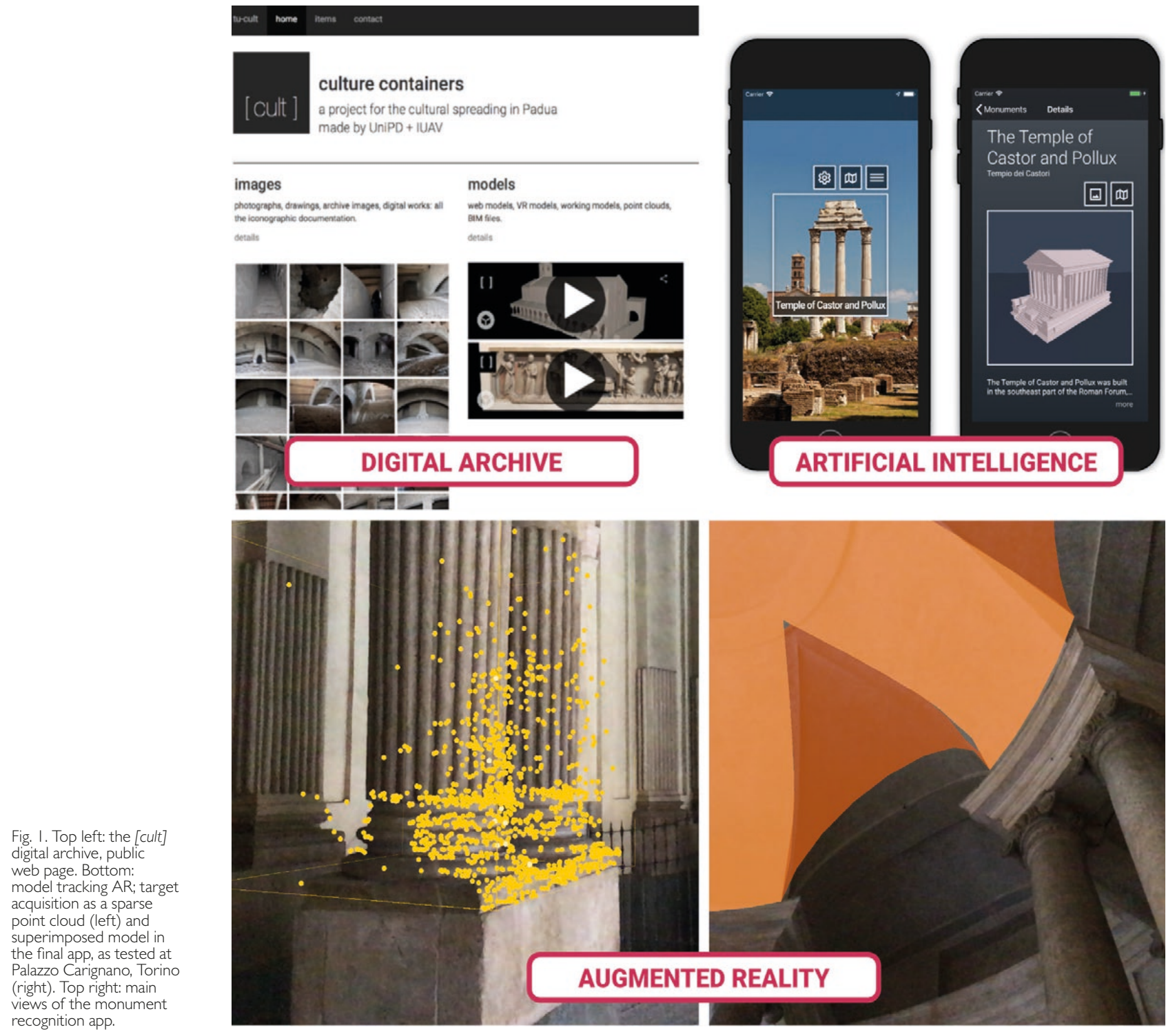

potential in the built environment [Pezzica et al. 2019]. In particular, we exploited a class of deep learning $(\mathrm{DL})$ algorithms that allows image recognition [Goodfellow, Bengio, Courville 20 I6]. Therefore, we have created an app that connects to the [cult] database and makes it accessible by pointing the camera of the mobile device at a monument (fig. I, top right). The Al system evaluates the frames recorded by the camera and proposes a forecast of the observed object. Then, the app shows information related to the identified monument, such as descriptive texts, images, interactive 3D models, the position on the map, and other data. For a first experiment, the system was applied in the Central Archaeological Area of Rome, where it allows the recognition of 46 monuments that are very different in shape, size, and state of conservation.

A significant step in building an Al system for image recognition is collecting the dataset for the training, that is, the process in which the parameters of the recognition algorithm are optimized for the required task. For this purpose, 50 to 100 photographs were collected for each monument to be recognized, choosing many different viewpoints on the object. The result is an engine that takes up about $13 \mathrm{MB}$ of disk space and can distinguish up to hundreds of objects (as an estimated magnitude) from every angle. The aspects that specifically concern the construction of the DL model are described in previous publications [Andrianaivo, D'Autilia, Palma 2019]. 


\section{Conclusions}

Technologies that interact with big-data risk replacing the work of interpreting the built environment and the relationship that binds us to it, be it about culture, fruition, or design. We must prevent a general understanding from being neglected. Theory and models are needed to achieve also detailed data-driven knowledge, to value documentation and collection efforts, and to clarify the purpose of these efforts. The presented series of knowledge access models intends to support the identification of some contexts and scopes that can benefit from experiments with AR and Al technologies. We investigated how digital techniques for spatial character recognition support a theoretical understanding and operational uses of the built form. On the one hand, we must strive to keep the landscape of these possible uses broad. On the other hand, even the schematic understanding of reality that tools produce - the categorizations, the standards - should be kept flexible. Otherwise, automatic recognition and classification become the well-known black box, that is, algorithms we accept the results of without questioning how these are produced or whether they can be adapted to changing needs. Finally, experiences suggest that, beyond the schemes we adopt, the tangible aspects of architecture remain a platform for effective knowledge sharing even in the virtuality-pervaded reality.

\section{Notes}

[I] The project was funded by the European program POR FSE 2014-2020 - Regione Veneto. A. Giordano was the principal investigator. L. Stendardo supervised the construction of the [cult] platform.

[2] ICEA department, UniPD (2017). [cult] - culture containers. http://cult.reload.dicea.unipd.it (I 5 March 202I).

[3] DCMI (202I). DCMl: Home. https://dublincore.org (I5 March 202I).

[4] In addition to the work of prof. Spallone and prof. Vitali, the research on the vaulted atria benefited from the collaboration of M.C. López González (Universitat Politècnica de València, project "Nuevas tecnologías para el análisis y conservación del patrimonio arquitectónico" funded by the Ministry of Science, Innovation and the University of Spain) and Ph.D. students G. Bertola, F. Natta and F. Ronco.

[5] Currently, some AR software such as Vuforia allow the tracing of very large environments based on CAD models or point clouds. See PTC (2020). Tools Overview | VuforiaLibrary https://library.vuforia.com/tools/overview.html (I 5 March 202 I).

[6] The team working on the project at FULL | The Future Urban Legacy Lab consisted of M. Robiglio, C. Casetti, F. Frassoldati, L.N. Andrianaivo e V. Palma. The project was carried out in partnership with R. d'Autilia.

\section{References}

Andrianaivo Louis Nantenaina, D'Autilia Roberto, Palma Valerio (2019). Architecture recognition by means of convolutional neural networks. In ISPRS - International Archives of the Photogrammetry, Remote Sensing and Spatial Information Sciences, XLII-2WI5, pp. 77-84.

Bekele Mafkereseb Kassahun, Pierdicca Roberto, Frontoni Emanuele, Malinverni Eva Savina, Gain James (20I8). A Survey of Augmented, Virtual, and Mixed Reality for Cultural Heritage. In Journal on Computing and Cultural Heritage, I I (2), pp. I-36.

Cecchini Cristina, Cundari Maria Rosaria, Palma Valerio, Panarotto Federico (2019). Data, Models and Visualization: Connected Tools to Enhance the Fruition of the Architectural Heritage in the City of Padova. In Marcos Carlos (ed.). Graphic Imprints. Cham: Springer, pp. 633-646.

Giordano Andrea (20 I7). Mapping Venice. From Visualizing Venice to Visualizing Cities. In Piga Barbara, Salerno Rossella (eds.). Urban Design and Representation. Cham: Springer, pp. I43-151.

Goodfellow lan, Bengio Yoshua, Courville Aaron (2016). Deep Learning. Cambridge: MIT Press.

Palma Valerio, Lo Turco Massimiliano, Spallone Roberta, Vitali Marco (20 I8). Augmented Iconography. AR applications to the fortified Turin in the Theatrum Sabaudiae. In Marotta Anna, Spallone Roberta (eds.). Defensive Architecture of the Mediterranean, IX, pp. I053-1060.

Palma Valerio, Spallone Roberta, Vitali Marco (2019). Augmented Turin baroque atria: AR experiences for enhancing cultural heritage. In ISPRS - International Archives of Photogrammetry, Remote Sensing and Spatial Information Sciences, XLII-2/W9, pp. 557-564.

Pezzica Camilla, Schroeter, J., Prizeman Oriel E., Jones Christopher B., Rosin, Paul L. (2019). Between Images and Built Form: Automating the Recognition of Standardised Building Components Using Deep Learning. In ISPRS Annals of Photogrammetry, Remote Sensing and Spatial Information Sciences, IV-2MW6, pp. I23-I32.

Spallone Roberta, Vitali Marco (20 I7). Star-shaped and Planterian Vaults in Turin Baroque Atria. Ariccia: Aracne.

Author

Valerio Palma, Dept. of Architecture and Design, Politecnico di Torino, valerio.palma@polito.it 
